\title{
Process Control of Drug Product Continuous Manufacturing Operations-a Study in Operational Simplification and Continuous Improvement
}

\author{
Joseph Medendorp $^{1} \cdot$ Sreedhar Shapally ${ }^{1} \cdot$ Derek Vrieze $^{1} \cdot$ Kelly Tolton ${ }^{1}$ \\ Accepted: 17 September 2020 / Published online: 29 September 2020 \\ (C) The Author(s) 2020
}

\begin{abstract}
Purpose The purpose of this manuscript is to demonstrate that implementation of gravimetric measurements provides the same assurance of product quality and process control as spectroscopic measurements (1) for control of drug content in a fixed-dose combination (FDC) tablet and (2) for identification of non-conforming material.

Methods A wet granulation continuous tableting line was used to make the FDC drug product batches. Comparative data was generated for ten batches using near-infrared (NIR) spectroscopy for core tablets, and gravimetric in-process control measurements (IPCs) applied to the ratio control of intra- and extra-granular blend (IG and EG). HPLC reference data were collected to further demonstrate uniformity at each stage of the production process, including IG, final blend, and core tablets. All possible sources of variation not directly detectable by the gravimetric measurements were considered and quantified.

Results The two IPC measurement techniques showed excellent agreement where both were within $2 \%$ of the target drug concentrations and within $2 \%$ of each other for the ten comparative batches. The NIR was more sensitive to material and process variations than the gravimetric IPCs; thus, it was more variable within and across batches. Gravimetric IPCs were demonstrated as an effective replacement for spectroscopic measurements for continuous tableting operations, capable of ensuring on target manufacturing and detection of non-conforming material.

Conclusions As pharmaceutical companies continue to push toward operational simplicity and sustainable manufacturing processes, soft-sensor and gravimetric controls as alternatives to their spectroscopic counterparts will be applied more broadly for process monitoring and control.
\end{abstract}

Keywords Gravimetric · PAT · Control strategy $\cdot$ Loss in weight $\cdot$ Continuous manufacturing $\cdot$ Regulatory

\section{Introduction}

\section{Process Description and Control Strategy}

The fixed-dose combination (FDC) tablet discussed in this paper is an immediate-release tablet for oral administration containing two active ingredients. One of the two active pharmaceutical ingredients (APIs) is a crystalline solid drug substance while the other is an amorphous spray-dried dispersion (SDD) intermediate. The FDC tablet contains $200 \mathrm{mg}$ of

Joseph Medendorp

Joseph_medendorp@vrtx.com

1 Vertex Pharmaceuticals, 50 Northern Avenue, Boston, MA 02210, USA crystalline API and $125 \mathrm{mg}$ of SDD and has a total target weight of $565.5 \mathrm{mg}$. The FDC tablets are manufactured using a continuous tableting line-25 (CTL-25, GEA, Belgium), which operates in a continuous mode from granulation to compression and in batch mode for initial powder blending and final tablet film coating. The CTL-25 is controlled by a qualified and automated process control system which provides for user access control, recipe management, recipe execution, material tracking, data logging, data visualization, and reporting for all integrated unit operations, including alarm logging for parameters outside their respective normal operating limits (NOR) and design space limits (DSL). The control scheme for the CTL25 includes configurable recipe-based alarm limits for the NORs and DSLs for process parameters, and all parameters are controlled relative to recipe set points by the automated control system (ACS). 
The manufacturing process is a wet granulation process, the details of which will be described below. A series of process control measurements are made at various stages of the manufacturing process including temperatures, pressures, flow rates, torque, and mass to ensure that the process runs in a state of control.

In early development, it was observed that the material transferred from one phase of the process (fluid-bed dryer) to the next phase of the process (mill) did not transfer completely under certain material and process-related conditions. With fixed targets for the intragranular blend (IG) and extragranular blend (EG) phase masses rather than proportional IG/EG phase mixing, this incomplete transfer of IG phase had the potential to create a ratio imbalance between the IG and EG content. This situation was addressed in three ways: (1) engineering controls to physically correct the incomplete transfer (reducing the frequency of occurrence); (2) addition of a load cell to quantify the amount of material present in the IG phase and ensure the appropriate addition of the EG phase, maintaining the target IG/EG ratio (reducing the severity in the event of an occurrence); and (3) addition of a near-infrared (NIR) core tablet in-process control measurement to confirm that core tablets were on target for the two APIs (improving the detectability of occurrence).

\section{Drivers for the Change-Lifecycles of Spectroscopic Methods}

\section{Advantages of Spectroscopic PAT}

Process analytical technology (PAT) is an important aspect of solid oral dosage manufacturing control strategies, including both non-spectroscopic (soft sensor) and spectroscopic-based PAT [1, 2]. Spectroscopic PAT, such as NIR and Raman, has been used successfully for real-time monitoring of uniformity and mixing, moisture content measurements in oral solid unit operations, physical form, and identification [3-10]. There are a number of advantages to using spectroscopic PAT. For example, these in-process control measurements can provide direct and chemically specific measurements of product attributes at key control points, ensuring that the manufacturing process is running in a state of control. Spectroscopic PAT can also be used to inform real-time release calculations, allowing users to release product immediately using representative data collected throughout the process, rather than collecting composite samples and sending to the laboratory for testing [11].

\section{Challenges for PAT Development, Implementation, and Maintenance}

While spectroscopic PAT can be used to advantage, it also carries disadvantages for a number of technical and industryspecific reasons, including (1) challenges to develop and implement and (2) a high degree of overhead and resources to maintain [12]. In order to ensure that spectroscopic PAT models can predict attributes of interest over the desired manufacturing ranges and across the multiple excipient and API lots used in commercial life cycle, using the most commonly employed regression-based techniques, spectroscopic calibration sets are designed to incorporate as much of this variation upfront as possible [13]. Therefore, to best anticipate how to keep models robust to future variation, a significant number of manufacturing runs, process, and material variations are required for inclusion or at least evaluation, in the calibration model. As new API/excipient sources are encountered over the course of commercial production, an evaluation of the impact to PAT models is necessary. For example, if an NIR model built to monitor chemical composition also happens to exhibit sensitivity to particle size differences between excipient suppliers; that may cause an artificial response in the concentration prediction. As such, this effect must be addressed in the design of the calibration set or the model parameters or must be controlled through operational and supply controls of input materials. From a global supply chain perspective, this is a complicating factor for the inclusion of spectroscopic PAT.

The high degree of overhead associated with PAT model maintenance and lifecycle management is also a complicating factor. Beyond the repeated evaluations required for spectroscopic PAT (second source addition, process changes, periodic parallel testing, etc.), each time a chemometric model is updated, there may be regulatory implications. Model maintenance updates are analogous to changes to analytical methods and therefore may require health authority approval prior to implementation. When spectroscopic PAT is included as an integral part of a manufacturing control strategy, there is a high level of scrutiny on the methods and the way they are maintained. Shi et al. present several recent advances toward the development of lower cost-of-ownership options for spectroscopic PAT methods, where either the time/ complexity for initial model development can be reduced or processes for long-term model maintenance can be simplified [14]. Similarly, with the adoption of ICH Q12 [15], regulatory mechanisms such as post-approval change management protocols (PACMP) and established conditions (ECs) have been designed and may be leveraged in conjunction with a company's pharmaceutical quality system (PQS) to reduce the regulatory efforts associated with PAT method lifecycle management. To the authors' knowledge, the full benefits of these recent technical and regulatory advances have not yet been fully realized. As such, there remains a real barrier to broad adoption of spectroscopic PAT methods across the industry, even when they provide effective solutions for process understanding, monitoring and control. 


\section{Advantages of Non-spectroscopic PAT}

Alternatively, non-spectroscopic PAT has been considered as an alternative means of in-process control and real-time release testing [16]. Process signatures, temperatures, and weight measurements can also provide operators an assurance of the state of control. In the present work, an at-line manual spectroscopic IPC measurement on core tablets in an oral solid dosage form manufacturing operation was successfully replaced with a non-spectroscopic pair of automated gravimetric IPCs which, taken together, accurately provided the chemical composition of the core tablets without the challenges and overhead associated with spectroscopic based PAT described above. Additionally, the automated gravimetric IPCs offered far greater operational simplicity as engineering controls in comparison with the manual procedural control provided by the NIR.

\section{Materials and Methods}

\section{Description of Continuous Manufacturing Equipment}

The manufacture of the FDC tablets used a continuous manufacturing process, starting with the introduction of pre-blended components and ending with core tablets, as illustrated in Fig. 1.

The FDC was manufactured with a wet granulation process, using the GEA continuous tableting line (CTL-25). The CTL-25 was controlled by the ACS which tracked the material using the concept of product keys (PK), or discrete units of material, which were tracked as they progressed through the process. The CTL-25 equipment employed a combination of fully continuous unit operations and continuous processing. Twin-screw wet granulation (TSWG) is an example of a truly continuous process, where the IG blend and the binder solution were continuously dosed into the granulator and granules continuously exit the granulator.

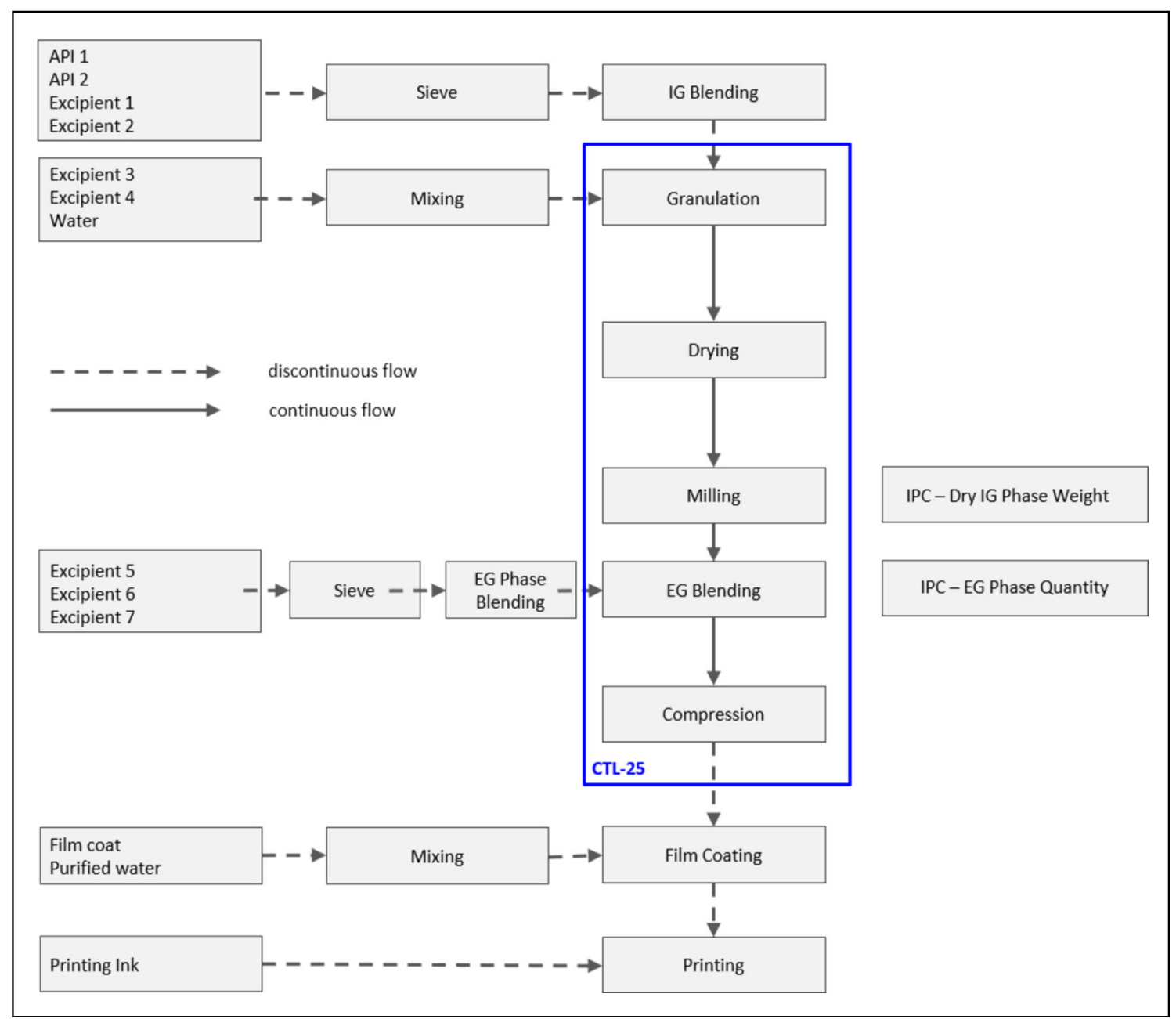

Fig. 1 Process flow diagram, illustrating the continuous manufacturing steps (inside the blue box with solid arrows) and discontinuous manufacturing steps (outside the blue box with dotted arrows) 
Following the TSWG step, the material was further processed as physically discrete PKs. These PKs remained physically separated until they are charged into the tablet press hopper, resulting in minimal intermixing and a relatively narrow residence time distribution across the full process train. The fluid-bed dryer (FBD) consisted of six identical drying cells that operated in parallel. Wet granules were charged into a drying cell for a defined time, the result of which was the functional definition of the PK (the material corresponding to one dryer cell). The size of a PK depended on the dryer cell filling time, the mass flow rate, and the target binder solution (explained in more detail in the "Description of Gravimetric In-Process Controls" section). Once a cell was filled, granules were charged sequentially into the next empty dryer cell. The granules in each filled cell were dried to a pre-defined drying temperature, at which point the contents of that cell were evacuated leaving the cell ready for the next filling cycle. The dried granules were conveyed via pneumatic transfer to the granule conditioning unit (GCU) for further milling.

The milled granules were subsequently blended with a preblend of EG excipients (EG phase) using a ribbon blender; and the resulting final blend was pneumatically transferred to a stack above the tablet press where it was gravity fed to a second ribbon blender. The final blend was gravity fed to the tablet press hopper and feed frame. The final blend was compressed to final a tablet applying a specified pre- and main compression force. As film coating and printing were batch processes and conducted separately from the continuous process, tablets from the CTL-25 press were collected in drums for transfer to the film coating and subsequent printing steps. If non-conforming material was detected, two segregation points were present in the CTL-25: after the GCU and after the tablet press.

The continuous manufacturing system used gravity for material transfer between unit processes where possible. Introduction of IG blend into the continuous system was by gravity flow from intermediate bulk containers (IBCs) into transfer chutes directly into the loss-in-weight (LIW) feeders. Sensors in the transfer chute ensured a sufficient volume of material is dispensed from the IBCs into the chute to supply the hopper of the LIW feeder for the next refill. Vibrators located on the transfer chutes and on the frame of the IBCs ensured consistent flow of material. A pneumatic conveying system was used to transfer wet granules and final blended granules to rotary tablet press.

LIW feeders are important for maintaining the desired mass flow for the process and setting the correct composition of final blend and thus are used in all locations where accurate material dosing was required. The use of LIW feeders has been published previously, and they have been used successfully for accurate continuous dispensing of pharmaceutical materials [1, 17]. Figure 2 displays the basic components of a LIW feeder. The LIW feeder uses an agitator that rotates through a powder bed to aid filling of screw flights with powder. The filled screw flights convey the powder through a

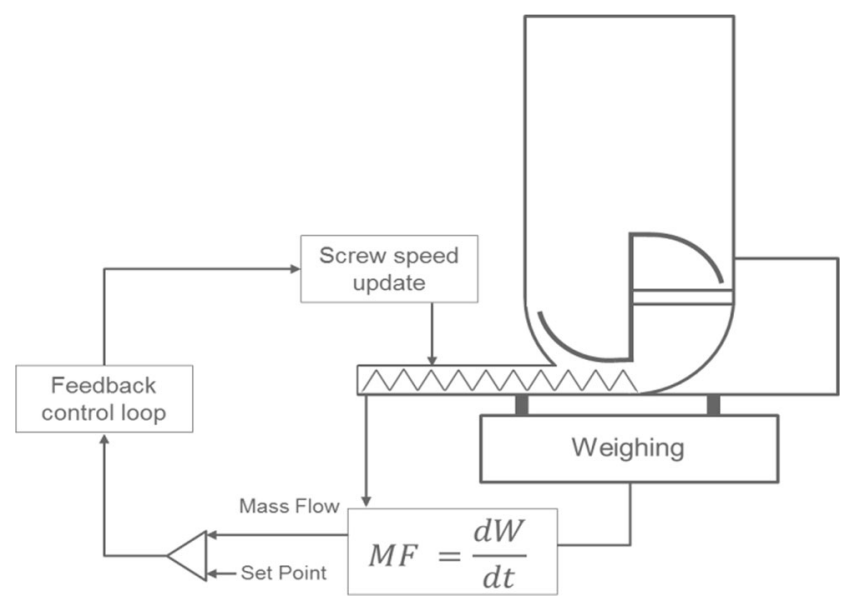

Fig. 2 Control schematic of a loss-in-weight feeder

barrel to the feeder outlet. Each LIW feeder uses an internal feedback control loop based on mass loss to continually control the screw speed in order to achieve a set point of target. Any discrepancy between the measured value and set point mass flow results in an adjustment of the screw speed to correct the mass flow.

\section{Spectroscopic In-Process Control}

The spectrometer used for the core tablet measurement was a Bruker Matrix I (Bruker Scientific, Billerica MA, USA) using a macro sample integrating sphere. The acquisition method was 32-scans at an 8-reciprocal centimeter resolution. A partial least squares (PLS) model was built for the detection of each active in a combination therapy tablet, the specifics of which are outside of the scope of this paper. Model specificity was ensured through a combination of experimental design and chemometric modeling practices. The NIR methods were built to incorporate sources of variation expected to occur in routine and non-routine commercial production (Table 1). Material attributes were included in the experimental designs, including multiple API lots, multiple excipient lots, specific API, and excipient lots that spanned the range of relevant material attributes (particle sizes, bulk densities, molecular weights, substitution ratios, moisture contents, etc.). Process and equipment were also included in the experimental design, including continuous and discontinuous equipment trains across multiple drug product manufacturing sites. And finally, spectrometer variation was included in the experimental design through the inclusion of multiple instruments over multiple preventative maintenance cycles (lamp changes, laser changes, wavelength calibrations, desiccant replacements). In addition to all of this, the actual chemical composition was varied around the target to ensure linearity and accuracy across the relevant concentration range.

The spectra included in each of the API models were deliberately selected from a larger pool in order to span the 
Table 1 Total variation considered for the calibration set alone

\begin{tabular}{ll}
\hline Parameter & Number included \\
\hline API 1 & 8 lots \\
API 2 & 11 lots \\
Excipient 1 & 3 lots \\
Excipient 2 & 5 lots \\
Excipient 3 & 9 lots \\
Excipient 4 & 5 lots \\
Excipient 5 & 4 lots \\
Excipient 6 & 5 lots \\
Excipient 7 & 5 lots \\
Drug product suppliers & 2 manufacturers \\
Equipment trains & 4 lines, continuous and discontinuous \\
Design spaces evaluated & Full range of design spaces \\
NIR Instruments & 3 instruments, all Bruker Matrix-I \\
\hline
\end{tabular}

claimed design spaces and material attributes described above. In some circumstances, it was the case that the inclusion of these attributes had a deleterious impact on the stated purpose of the model, namely, to accurately measure the chemical composition of the API in the core tablet. The total material and process variation represented in the calibration set alone is illustrated in Table 1.

The model was calibrated on a set of known samples, tested on an independent set of known samples that also spanned the range of concentrations and process parameter ranges, then validated against another set of independent samples. The elements of the validation were consistent with a typical ICH validation. Pure component API spectra were compared with the PLS loading vectors to ensure model specificity and that the loading vectors were consistent with the wavelength regions most highly correlated to the API features.

The implementation of the NIR method required at-line testing of a tablet from each PK, corresponding to approximately every $3 \mathrm{~min}$ of production. In the event of failing model diagnostics, additional tablets were scanned to confirm the original results. In the event of measurements that failed an IPC limit, the PK was rejected from the batch. The automation of the NIR method was handled in synTQ 4.0 (Optimal Ltd., Bristol UK), which presented a simple user interface to the operators for ease of use. Each PM cycle for the Bruker method resulted in a necessary update to the synTQ orchestration, along with all the requisite accompanying documentation for a GMP installation. Additionally, there was a parallel testing requirement (both annual and event driven) where the NIR results were required to comply with the offline reference method within predefined acceptance criteria. Each time second source APIs or excipient suppliers were qualified or elements of the manufacturing process were changed, the potential impact to the PAT method needed to be assessed. If impact was expected, the model needed to be updated in advance of production, consistent with the highly regulated pharmaceutical manufacturing environment. All these elements were factors in the decision to consider replacement of the NIR.

\section{Description of Gravimetric In-Process Controls}

The load cell is a gravimetric measurement device designed to identify and segregate material on the basis of weight of the dried, milled granules (net weight), as well as by the addition of the appropriate quantity of EG phase to the granules (Fig. 3). Milled granules are discharged from the mill into the load cell where the weight of each discrete unit of mass (PK) is verified against the theoretical weight of the PK based on the known IG mass-flow rate, binder solids concentration, and the dryer cell fill time.

At an IG blend feed rate of $15.6 \mathrm{~kg} / \mathrm{h}, 4.3 \%$ binder solids and 3-min dryer fill-time, the expected weight of the dried, milled granules is $815 \mathrm{~g}$.

A recipe tolerance of $\pm 20 \%$ (of the target IG phase content) was used during production to determine the rejection criterion for IG blend weight (actual weight by load cell-estimated weight from LIW Feeder \#1). Therefore, quantities of dried, milled granules outside of $652 \mathrm{~g}$ to $978 \mathrm{~g}$ were marked for segregation. Once weighed, the PK was discharged from the load cell into Blender \#1. At this point, LIW Feeder \#2 delivered the appropriate amount of EG phase to the dried, milled granules in the proportion of $22.9 \%$ to the IG phase weight (from the actual load cell measurement), bringing the PK to the correct composition. A recipe

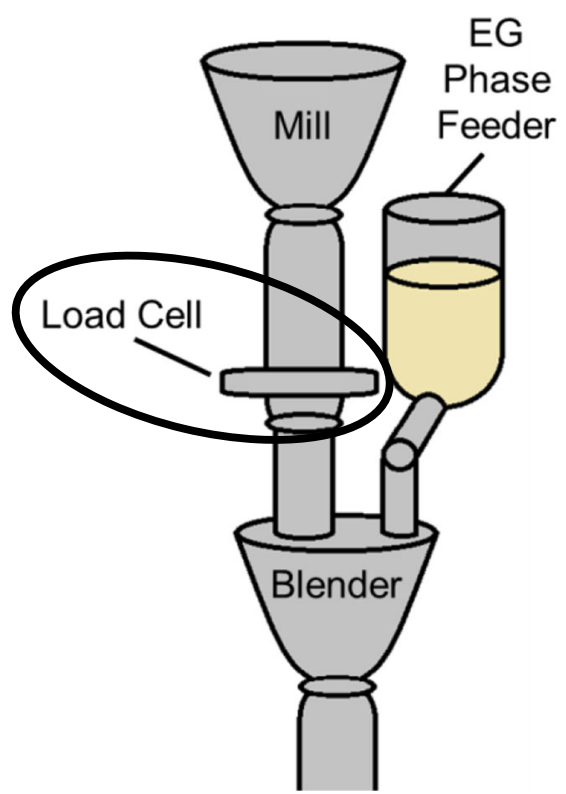

Fig. 3 Schematic representation of the load cell and where in the CTL-25 it is installed 
tolerance of $\pm 13 \%$ (of the target EG phase content) was used during production to determine the rejection criterion for the amount of EG phase dosed. The result of these two load cell measurements and segregation criteria was that dried, milled granules were always dosed to within \pm 20 tolerance of the expected weights, and EG phase was always dosed to within $\pm 13 \%$ of the target. The theoretical impact of these tolerances and additional sources of variability on tablet assay will be presented below.

The \% label claim for the load cell was calculated using the following equations:

API $1 \% \mathrm{LC}=\frac{100 \times \mathrm{A} \times\left(\mathrm{IG}_{\text {full }}-\mathrm{IG}_{\text {empty }}\right)}{\mathrm{B} \times\left(\mathrm{IG}_{\text {full }}-\mathrm{IG}_{\text {empty }}+\mathrm{EG}_{\text {Dosed }}\right)}$,

where

A target $\%$ of API 1 in the IG phase

B target \% of API 1 in the total blend (final blend composition is $97.1 \%$ of the coated tablet composition)

API $2 \% \mathrm{LC}=\frac{100 \times \mathrm{C} \times\left(\mathrm{IG}_{\text {full }}-\mathrm{IG}_{\text {empty }}\right)}{\mathrm{D} \times\left(\mathrm{IG}_{\text {full }}-\mathrm{IG}_{\text {empty }}+\mathrm{EG}_{\text {Dosed }}\right)}$,

where

C target $\%$ of API 2 in the IG phase

D target \% of API 2 in the total blend (final blend composition is $97.1 \%$ of the coated tablet composition).

\section{Results and Discussion}

\section{Load Cell Assumptions and Sources of Variation}

In order to switch from the direct analytical IPC measurement of API concentration to gravimetric IPCs, it was necessary to consider all possible sources of variation not directly detectable by the LIW feeder/load cell measurements. It is a prerequisite that the load cells are maintained under a preventive maintenance program and checked daily for accuracy using certified weight standards. Additional factors for consideration included precision in material dispensing, the assurance of uniformity at both the granule and the final blend stage, the evaluation of moisture content introduced throughout the process, and the cumulative impacts of these factors on the calculated blend potency. Each of these will be discussed in the upcoming sections.

\section{Uniformity of Intragranular Blend, Final Blend, and Core Tablets}

In the present section, uniformity data will be presented for a single batch from each of the stages of the process by the HPLC reference method (for blends and core tablets) and/or by the NIR method (core tablets). The active IG blending operation was performed using a 500-1 stainless steel IBC blender, prepared at a commercial scale. IG blend uniformity was measured from early process development through process validation. IG blending was for a fixed number of revolutions, and blend uniformity was evaluated by collecting thief samples from a 10-point stratified sampling plan from each

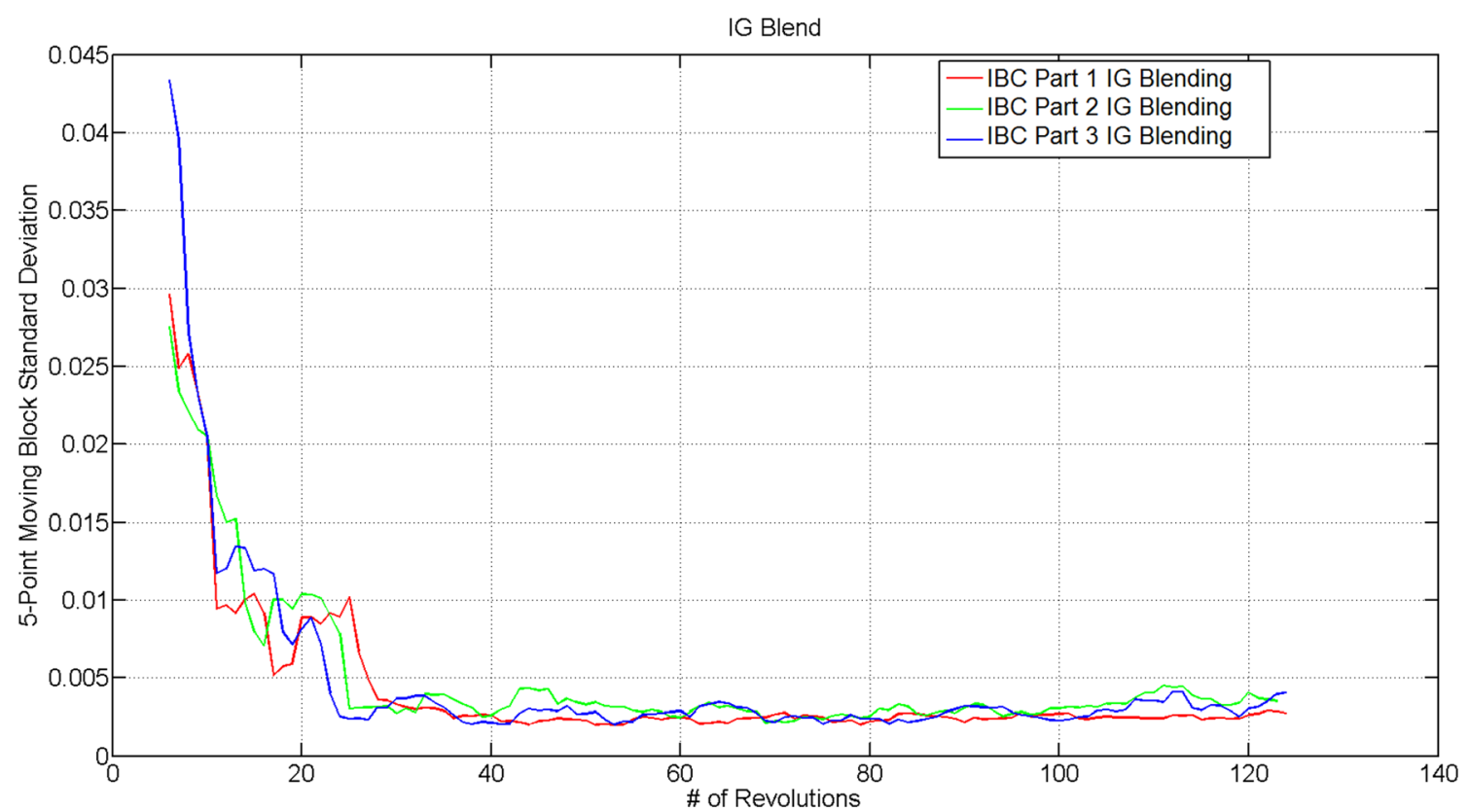

Fig. 4 Online IG blend uniformity measurements using a 5-point moving block standard deviation 
Table 2 Active IG blend uniformity testing results from three representative IBCs

Process validation batch
Acceptance criteria: average content, $90.0-110.0 \%$ for API 1\&2; RSD, NMT 5.0\%

All individual values are $\pm 10 \%$ of the mean

\begin{tabular}{|c|c|c|c|c|c|}
\hline \multicolumn{2}{|l|}{ Bin 1} & \multicolumn{2}{|l|}{ Bin 2} & \multicolumn{2}{|l|}{ Bin 3} \\
\hline \multicolumn{2}{|c|}{ Pre-lubrication } & \multicolumn{2}{|c|}{$15 \mathrm{~min} / 8 \mathrm{rpm}$} & \multicolumn{2}{|c|}{$15 \mathrm{~min} / 8 \mathrm{rpm}$} \\
\hline API $1 \%$ LC & API $2 \%$ LC & API $1 \%$ LC & API $2 \%$ LC & API $1 \%$ LC & API $2 \%$ LC \\
\hline 102.0 & 99.8 & 101.0 & 99.4 & 101.0 & 99.8 \\
\hline 0.8 & 0.7 & 1.0 & 1.2 & 0.3 & 0.4 \\
\hline Yes & Yes & Yes & Yes & Yes & Yes \\
\hline
\end{tabular}

IBC. The results confirmed the active ingredients were distributed uniformly throughout the IBC and that the blending process was adequate to ensure blend homogeneity. In addition to the thief sampling, the IG blend process was also monitored using an online NIR probe (Prozess 601, Chesterfield MO, USA) fitted to a custom-built IBC lid. The moving block standard deviation illustrated in Fig. 4 confirmed that the bin blending process produced a homogeneous IG blend by 30 revolutions, and the data in Table 2 confirm that the IG blend is well blended at the end of the blending operation.

In addition to the IG blend uniformity, final blend was also sampled from the process in a stratified fashion. Seven samples were collected and tested from the start of the run (every 5th PK for $35 \mathrm{PKs}$ ), seven samples were collected from the middle of the run (every 5 th PK for $35 \mathrm{PKs}$ ), and seven samples were collected from the end of the run (every 5th PK for $35 \mathrm{PKs}$ ). The final blend uniformity results are presented in Table 3, confirming that the final blend was well blended and was at the target potency.

Three core tablet samples were collected and tested at the lab from the start of the run (every 5 th PK for 35 PKs), seven samples were collected from the middle of the run (every 5 th PK for $35 \mathrm{PKs}$ ), and seven samples were collected from the end of the run (every 5 th $\mathrm{PK}$ for $35 \mathrm{PKs}$ ). The core tablet results are presented in Table 4 and show good content uniformity, further confirming that the final blend was well blended and was at target potency. Core tablets were also sampled at 3-min intervals for NIR analysis throughout the run. A single tablet was tested at each timepoint by NIR, the results of which are also presented in Table 4.

In order to support the case that gravimetric IPCs could replace the direct spectroscopic measurement, it was a prerequisite that the blend was uniform at each stage of the manufacturing process. The results presented in the tables above indicate that this prerequisite was met and that the loss-in-weight feeders accurately dispensed the material, the blenders properly blended the material, and that the mixing present in the line ensured the tablet blend remained uniform throughout the manufacturing process.

\section{Material Dispensing Precision}

The tolerance on material dispensing matches the precision on the balance. For example, if the precision of the balance is 2 decimal places on a kilogram scale, and the target drug substance weight is $106.11 \mathrm{~kg}$, the range of possible weights is $106.105-106.114 \mathrm{~kg}$. When the assay calculation is performed from a theoretical batch composition using the range above, the impact of dispensing accuracy can be seen in the second decimal place (i.e., $100.00 \%$, versus $100.01 \%$ ). The assay method rounds to a single decimal place; therefore, this difference is negligible.

\section{Moisture Content}

Without the direct measure of API concentration in a core tablet, fluctuations in moisture content could impact the assay as well. There are many different factors to consider for moisture, including moisture content in the APIs and excipients at dispensing, moisture content added during granulation and subsequently removed in the fluid-bed dryer from the granules, and the amount of EG phase delivered to make the final blend. Each of these scenarios was considered for the calculations shown in Table 5.

The moisture values used for these calculations for IG blend (low and high moisture) were the sum of the equilibrium moisture contents for each formulation component at $35 \% \mathrm{RH}$ (1.6\% moisture total in the IG blend) and $60 \% \mathrm{RH}(2.8 \%$ moisture total in the IG blend), respectively. The moisture values used for the fluid-bed drying calculation were a decrease in moisture from the IG stage to $1.0 \%$, and an increase

Table 3 Final blend - blend uniformity testing by HPLC

\begin{tabular}{lll}
\hline API & API 1\%LC & API 2 \% LC \\
\hline Mean & 100.2 & 99.0 \\
$\%$ RSD & 0.5 & 0.6 \\
Complies with acceptance criteria & Yes & Yes \\
\hline
\end{tabular}


Table 4 Core tablets content uniformity by lab

\begin{tabular}{|c|c|c|c|c|}
\hline \multirow[b]{2}{*}{ API } & \multicolumn{2}{|c|}{ HPLC \% LC } & \multicolumn{2}{|c|}{ NIR \% LC } \\
\hline & API & API 2 & API 1 & API 2 \\
\hline Mean & 99.1 & 99.3 & 100.3 & 99.1 \\
\hline$\%$ RSD & 1.1 & 1.2 & 0.9 & 0.7 \\
\hline Complies with acceptance criteria & Yes & Yes & Yes & Yes \\
\hline
\end{tabular}

to $3.2 \%$ moisture (to match the dried granule IPC limit from development). Likewise, the low and high moisture values were the sum of equilibrium moisture values at $35 \%$ and $60 \% \mathrm{RH}$ for the formulation components in the EG phase. These values were expected to be extreme approximations for the amount of water present or introduced at each stage. Using these moisture contents, the EG phase was added high and low according to the \pm 13 tolerance limit, and the total impact of moisture on assay was calculated. Moisture content plays the most significant role for high assay under the following conditions: high IG moisture/low FBD moisture/EG $<$ target. When IG moisture is high, excipients contain a relatively higher amount of moisture than the API, so API is more concentrated relative to dry solids content. When the granules are highly dried, it further concentrates the API concentration relative to the total mass of the EG phase. If EG is subsequently dosed on the lower end of the tolerance, it further concentrates the API concentration in the final blend since API is present only the IG phase. Conversely, moisture content plays the most significant role for low assay with the opposite situation: low IG moisture/high FBD moisture/EG $>$ target. With low IG moisture, the API solids concentration is closer to target relative to the excipients in the IG blend, on a dry basis. With high moisture after drying, the effective API concentration is lower in the granules. Compounded with EG delivered $>$ target, that further reduces the effective API concentration in the final blend. These calculations illustrate that even at the very extremes for moisture content present in the excipients or introduced in the granulation, compounded with the extremes of the EG LIW feeder tolerance, theoretical API assay would still be within 5\% of target for all but one scenario. Therefore, moisture is unlikely to negatively impact the API assay in routine production.

\section{Comparative Data-Gravimetric vs. Spectroscopic}

Figures 5 and 6 illustrate the performance of the gravimetric IPC measurements versus the performance of the NIR over the course of these 10 batches. The trends in both mean API content as well as variability are consistent between the two measurements. The numbers are presented in Table 6 for the mean and difference in content for API 1 and API 2 as measured by NIR compared with the mean by gravimetric analysis.

For a single batch shown in Fig. 7, HPLC testing of samples from beginning, middle, and end of the batch is also shown along with gravimetric and NIR results. This figure shows very close agreement between the load cell, the NIR, and the offline HPLC sampling.

\section{Process Capability and Justification of Limits}

For dried, milled granules, the $\pm 20 \%$ IG tolerance resulted in an acceptable range from 652 to $978 \mathrm{~g}$. The measured weight from the load cell ensured that LIW Feeder \#2 dosed the appropriate amount of EG material as calculated from the amount of IG material weighed,

Table 5 Introduction of moisture at various stages of process in the impact on $\% \mathrm{LC}$

\begin{tabular}{|c|c|c|c|c|}
\hline & & EG weight & API 1 assay & API 2 assay \\
\hline \multirow[t]{3}{*}{ Low IG moisture (1.6\% before granulation) } & \multirow{3}{*}{$\begin{array}{l}\text { Low FBD moisture } \\
\text { (Dried to } 1 \% \text { ) }\end{array}$} & Target $+13 \%$ & 97.8 & 96.8 \\
\hline & & Target $-13 \%$ & 102.7 & 101.6 \\
\hline & & At target & 100.2 & 99.2 \\
\hline \multirow{3}{*}{$\begin{array}{l}\text { Low IG moisture } \\
\text { (1.6\% before granulation) }\end{array}$} & \multirow{3}{*}{$\begin{array}{l}\text { High FBD moisture } \\
\text { (Dried to } 3.2 \% \text { ) }\end{array}$} & Target $+13 \%$ & 95.7 & 94.7 \\
\hline & & Target $-13 \%$ & 100.4 & 99.4 \\
\hline & & At target & 98.0 & 97.0 \\
\hline \multirow{3}{*}{$\begin{array}{l}\text { High IG moisture } \\
\text { ( } 2.8 \% \text { before granulation) }\end{array}$} & \multirow{3}{*}{$\begin{array}{l}\text { Low FBD moisture } \\
\text { (Dried to } 1 \% \text { ) }\end{array}$} & Target $+13 \%$ & 99.0 & 97.3 \\
\hline & & Target $-13 \%$ & 103.9 & 102.1 \\
\hline & & At target & 101.4 & 99.6 \\
\hline \multirow{3}{*}{$\begin{array}{l}\text { High IG moisture } \\
\text { ( } 2.8 \% \text { before granulation) }\end{array}$} & \multirow{3}{*}{$\begin{array}{l}\text { High FBD moisture } \\
\text { (Dried to } 3.2 \% \text { ) }\end{array}$} & Target $+13 \%$ & 96.8 & 95.1 \\
\hline & & Target $-13 \%$ & 101.6 & 99.8 \\
\hline & & At target & 99.1 & 97.4 \\
\hline
\end{tabular}




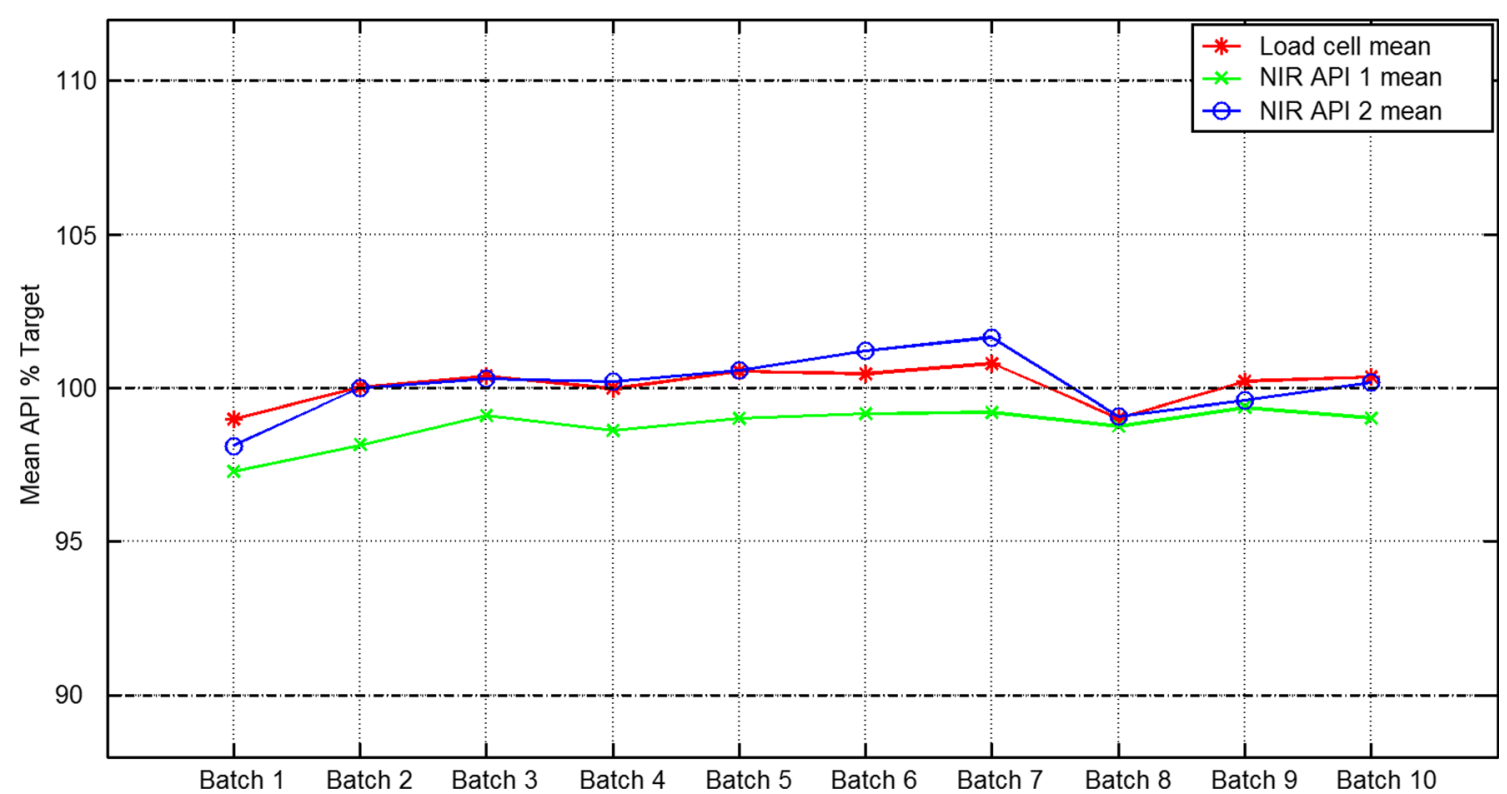

Fig. 5 Mean API \% of target for 10 representative batches, load cell compared with NIR

by maintaining the same EG/IG ratio of $22.9 \%$. The tolerance limits on the EG phase dosing were set to \pm $13 \%$ of the target weight. The calculations shown below and presented in Table 7 demonstrate that the $\pm 13 \%$ tolerance on EG phase dosing amounted to a $2.5 \%$ change in API \% label claim. In other words, assuming a uniform IG blend, the CTL-25 was programmed to physically deliver final blend within $2.5 \%$ of target. As such, the tolerance limits on the load cell were appropriate to control the process and the product.

With the same calculation used to calculate Table 7, it can be shown that even with an EG phase tolerance at $\pm 25 \%$, API concentration would also meet $95-105 \%$ of the target final blend concentration. By setting the limits at \pm 13 , there is still an allowance for normal process variability, but there is assurance that blend composition will be at target. As described, the NIR IPC was originally implemented to eliminate the risk of passing a PK where material transfer from the fluid-bed dryer to the GCU hopper was incomplete, which following the addition of EG phase would have the incorrect IG/EG ratio. However, with the implementation of the load cell IPCs, risk of incomplete PK transfer from the dryer is effectively eliminated, and the risk of producing final blend with the incorrect IG/ EG ratio is minimal.

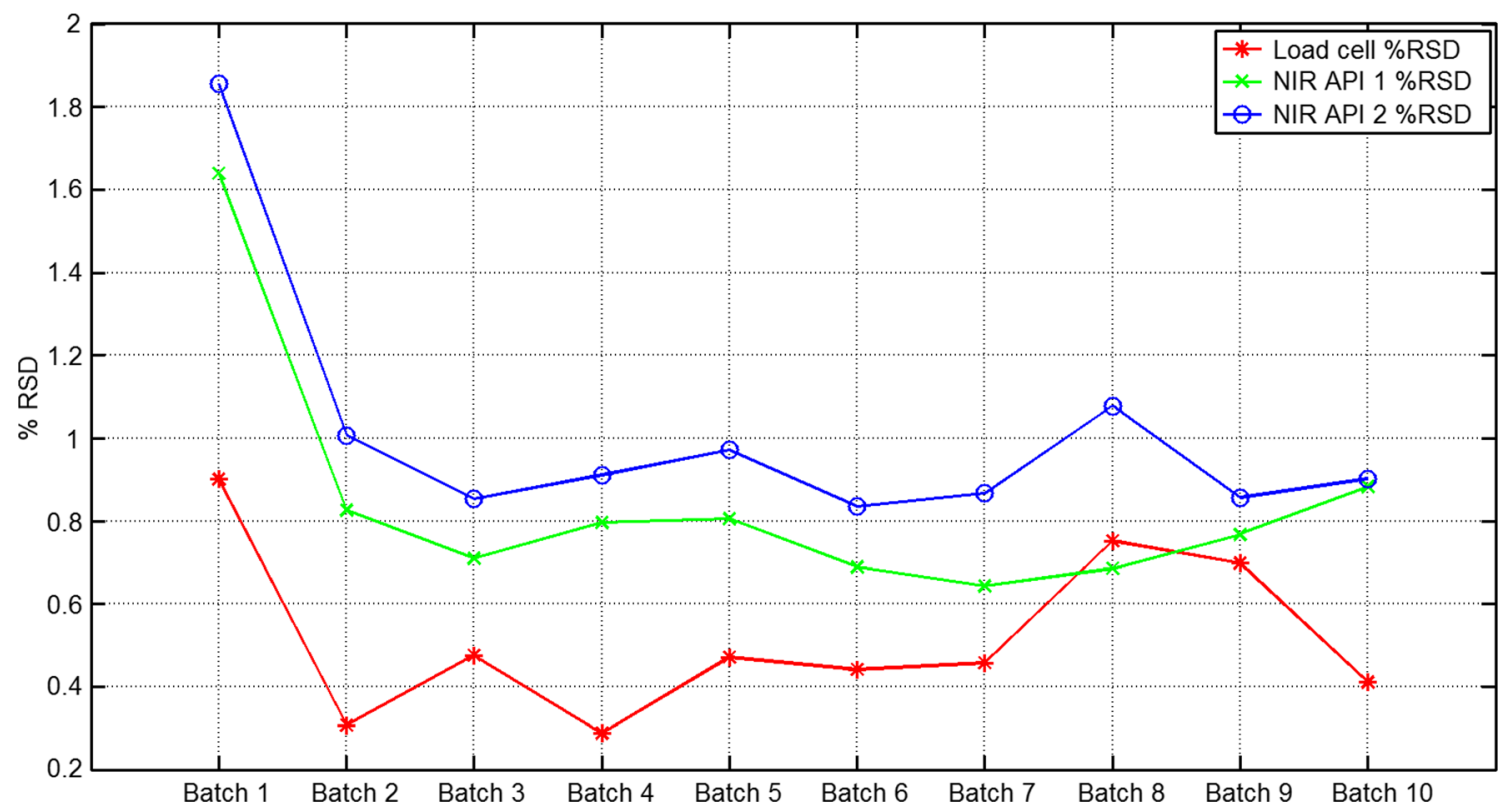

Fig. 6 Mean API \% RSD for 10 representative batches, load cell compared with NIR 
Table 6 Statistical comparison of load cell with NIR results

\begin{tabular}{llc}
\hline & Parameter & Result \\
\hline Load cell and API 1 & Min (\% difference) & 0.25 \\
& Max (\% difference) & 1.87 \\
& Mean (\% difference) & 1.3 \\
Load cell and API 2 & Min (\% difference) & -0.85 \\
& Max (\% difference) & 0.87 \\
& Mean (\% difference) & -0.01 \\
\hline
\end{tabular}

For the PKs that were within the tolerance, the distribution of IG weights is presented in Fig. 8 for 10 batches. PKs that did not meet the IG tolerance were segregated from the process as non-conforming material and thus were not included in the figure or the analysis. The initial IG weight target did not factor in the contribution of the binder solids; thus, IG weights were reported here as 3-4\% higher than target on average. Even when offset by the missing binder solids, the IG weights were still within the $\pm 20 \%$ tolerance. Since this mass was indicative of any potential problems with incomplete material transfer, any PKs were automatically removed from the process when they did not conform to the $\pm 20 \%$ tolerance.

Similarly, the distribution of EG weights is presented in Fig. 9 relative to the tolerance of $\pm 13 \%$ for those PKs that met the tolerance. Two batches had higher than expected variability for EG phase dosing and were slightly higher than targeted for proportional dosing. Both examples had known root causes, easily tracked to mechanical interference with the load cell.
Table 7 Tolerance limits for IG and EG-phase dosing

\begin{tabular}{lll}
\hline & LL $(g)$ & UL $(g)$ \\
\hline IG $(g)$ & 652 & 978 \\
Target EG $(g)$ & 149 & 223 \\
Target EG $(g)-13 \%$ & 130 & 194 \\
Target EG $(g)+13 \%$ & 168 & 253 \\
\%LC at Target EG $(g)$ & 100.0 & 100.0 \\
\% LC at Target EG $-13 \%$ & 102.5 & 102.5 \\
\%LC at Target EG $+13 \%$ & 97.6 & 97.6 \\
\hline
\end{tabular}

\section{Impact of Disturbances/Perturbations}

In pharmaceutical manufacturing operations, it is important to understand the types of process disturbances that could occur, the factors that can be responsible for how they occur, and to gauge the potential impact to product quality that disturbances could have should they occur. Materials and process risk assessments were used to identify and quantify the level of risk. The outcome of these risk assessments was to inform the experimental design and the set of mitigating engineering and process controls. For this manufacturing process, the two most significant risks identified were inconsistent material feeding to the twin-screw wet granulator and incomplete material transfer from the fluid-bed dryer to the granule conditioning unit. Armed with this information, it was possible to design engineering and procedural controls to ensure that these disturbances (1) did not occur in routine manufacturing and (2) were readily detected if they did occur, so the impact of the disturbance could be assessed and, if necessary, the material removed from the process.

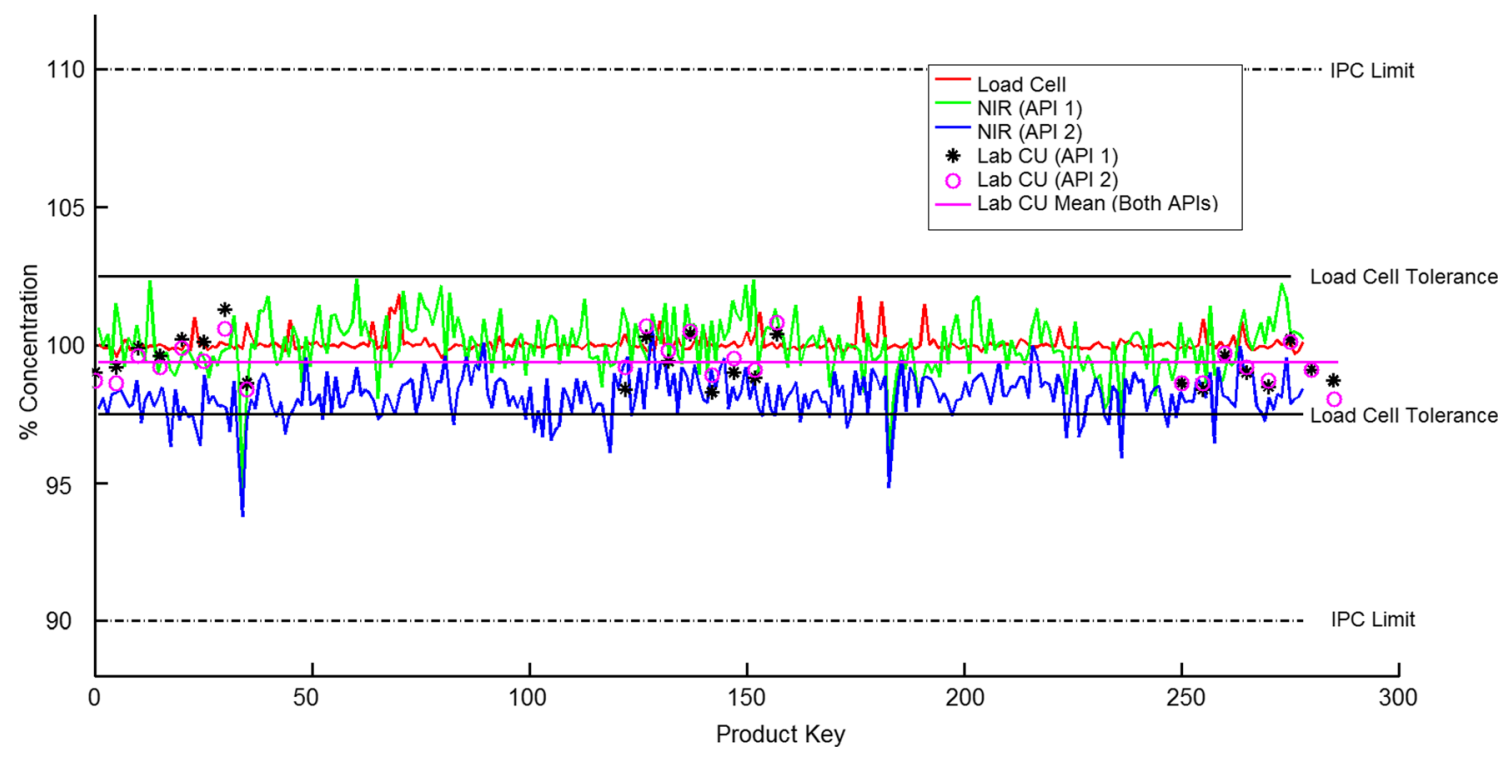

Fig. 7 Overlay example with load cell, NIR, and lab HPLC data 


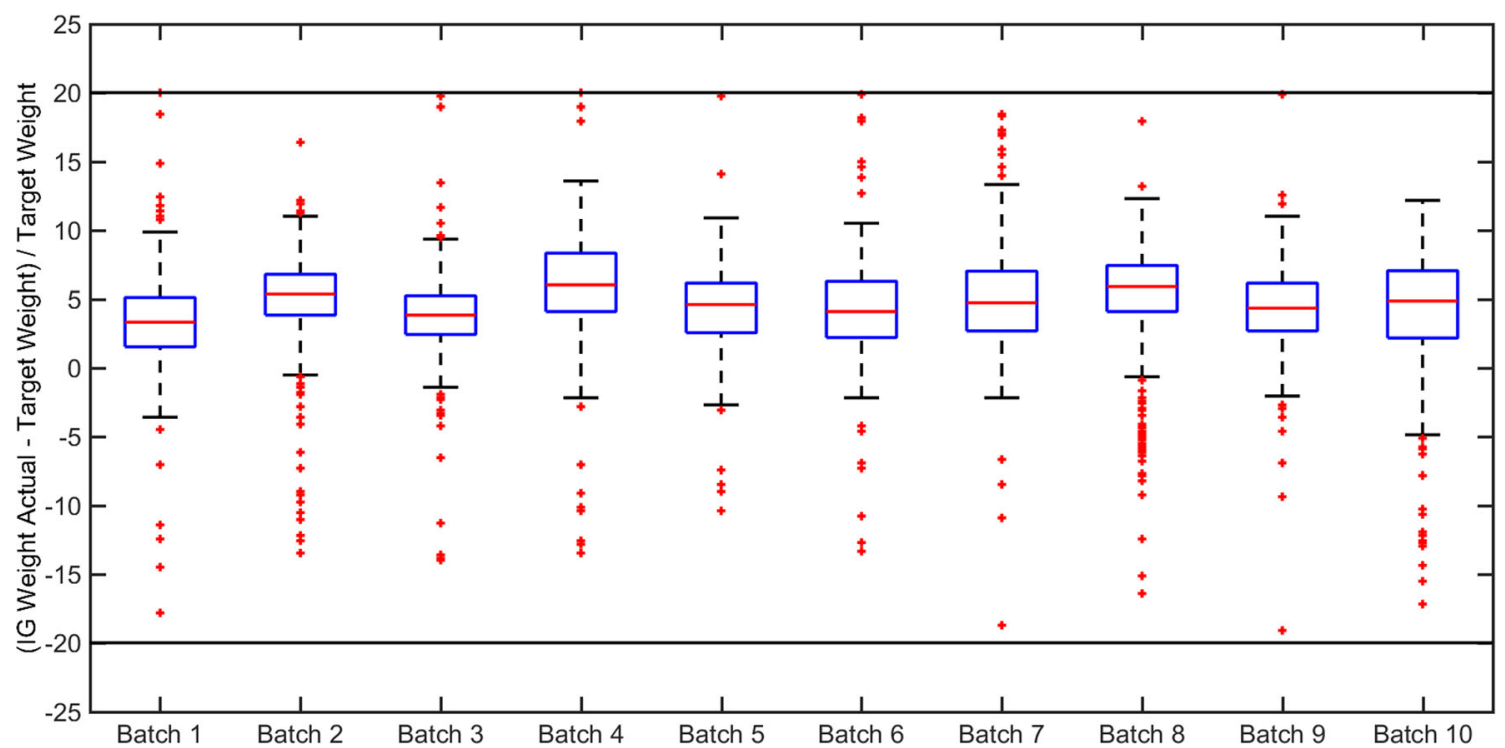

Fig. 8 IG weights presented relative to the $\pm 20 \%$ tolerance limit

\section{Regulatory Impact, Global Approval, and Global Supply}

A post approval change was submitted describing the replacement of the NIR spectroscopic IPC with two gravimetric IPCs, which collectively provided equivalent control of the tablet API concentration, reducing the risk of producing final blend with the off-target IG/EG ratio in an operationally simpler way. This control strategy change was not a straightforward replacement of a test since the two methods have different inputs and measure the in-process material potency at different points in the continuous manufacturing process. Also, changes to an IPC for active content can have a high potential to impact product quality. Therefore, health authority approval was necessary prior to implementation.
Three health authorities (FDA, EMA and Health Canada) issued questions regarding the change. Common requests included a comparison of gravimetric to NIR data, further justification of the IPC limits for IG and EG phase weights, sampling frequency, and product segregation procedures. All requested data was provided. Perhaps the most compelling data submitted was a plot of the IG and EG phase IPC values for 10 consecutive batches (Figs. 5 and 6). This allowed the reviewer to see that the IPC acceptance limits were determined not only by assessing impact to the final product but also through process capability. All questions were resolved with one round of questions from the three health authorities, and this change was also approved in Australia, Israel, and Switzerland.

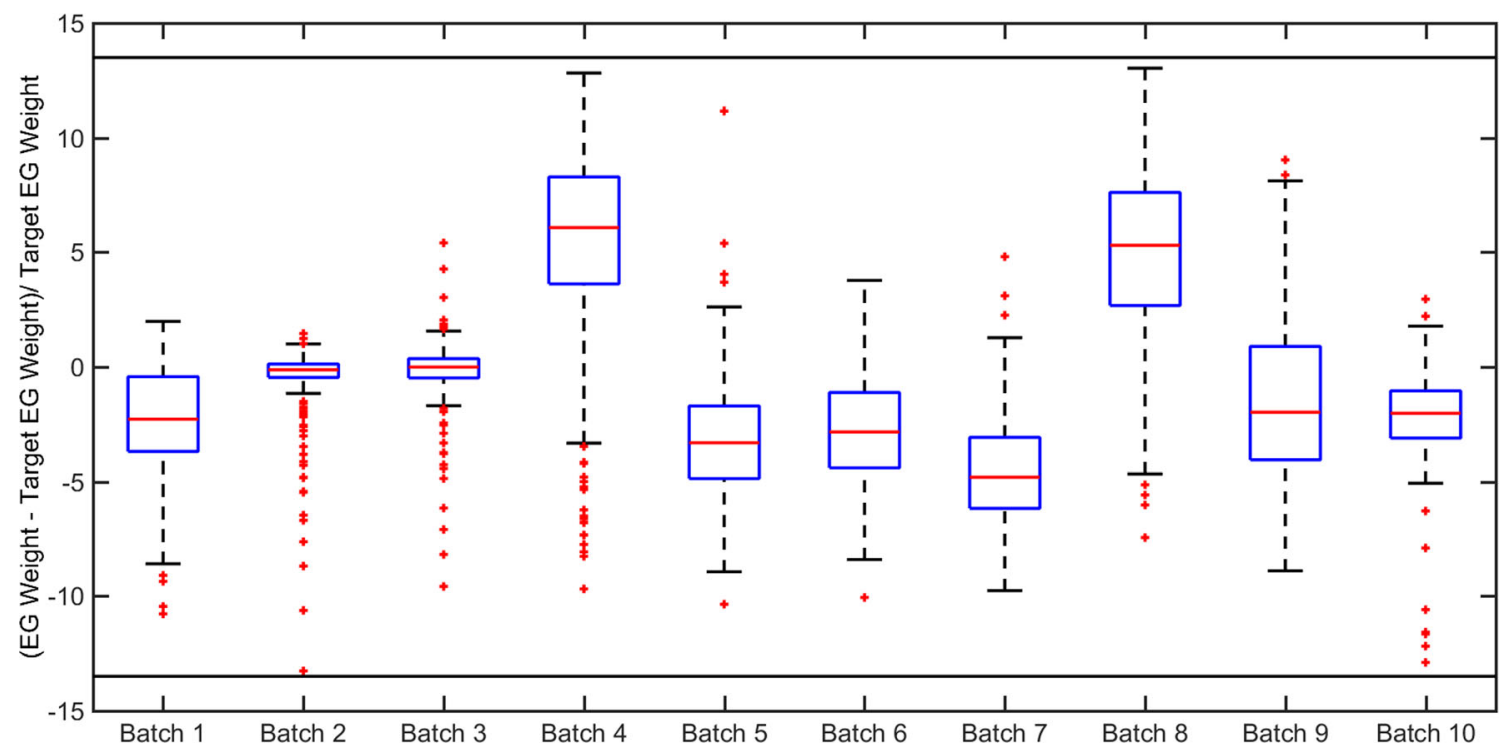

Fig. 9 EG weights presented relative to the $\pm 13 \%$ tolerance limit 


\section{Conclusions}

The results of the LIW feeder/load cell IPC measurements and segregation criteria are that dried milled granules for passing PKs are always dosed to within \pm 20 tolerance of the expected weights, and EG phase is always dosed to within $\pm 13 \%$ of the target, and the API concentration is effectively maintained within $\pm 2.5 \%$ of target. There was excellent agreement between the two techniques for the measurement of API \% concentration. As such, the gravimetric IPCs effectively control API content in final blend for the CTL-25. Additionally, possible sources of variability detectable by NIR that may not be detectable by gravimetric analysis (material dispensing precision and moisture content) were assessed and were considered well controlled for routine production. The two gravimetric IPCs were demonstrated to be an effective replacement for the previous NIR IPC for the measurement of API concentration, demonstrating comparable process control while significantly reducing the operational and procedural complexity for a continuous manufacturing drug product process.

\section{Compliance with Ethical Standards}

Conflicts of Interest The authors declare that they have no conflicts of interest.

Open Access This article is licensed under a Creative Commons Attribution 4.0 International License, which permits use, sharing, adaptation, distribution and reproduction in any medium or format, as long as you give appropriate credit to the original author(s) and the source, provide a link to the Creative Commons licence, and indicate if changes were made. The images or other third party material in this article are included in the article's Creative Commons licence, unless indicated otherwise in a credit line to the material. If material is not included in the article's Creative Commons licence and your intended use is not permitted by statutory regulation or exceeds the permitted use, you will need to obtain permission directly from the copyright holder. To view a copy of this licence, visit http://creativecommons.org/licenses/by/4.0/.

\section{References}

1. Rehrl J, Karttunen AP, Nicolai N, Hörmann T, Horn M, Korhonen $\mathrm{O}$, et al. Control of three different continuous pharmaceutical manufacturing processes: use of soft sensors. Int J Pharm. 2018;543:60-72.

2. Huang J, Romero-Torres S, Moshgbar M. Practical considerations in data pre-treatment for NIR and Raman spectroscopy. Am Pharm Rev. 2010;13(6):116-27.
3. Bogomolov A, Mannhardt J, Heinzerling O. Accuracy improvement of in-line near-infrared spectroscopic moisture monitoring in a fluidized bed drying process. Front Chem. 2018;6:388.

4. Fonteyne M, Vercruysse J, De Leersnyder F, Van Snick B, Vervaet $\mathrm{C}$, Remon JP, et al. Process analytical technology for continuous manufacturing of solid-dosage forms. TrAC Trends Anal Chem. 2015;67(Supplement C):159-66.

5. Esmonde-White K, Cuellar M, Uerpmann C, Lenain B, Lewis I. Raman spectroscopy as a process analytical technology for pharmaceutical manufacturing and bioprocessing. Anal Bioanal Chem. 2017;409(3):637-49.

6. Järvinen K, Hoehe W, Järvinen M, Poutiainen S, Juuti M, Borchert $\mathrm{S}$. In-line monitoring of the drug content of powder mixtures and tablets by near-infrared spectroscopy during the continuous direct compression tableting process. Eur J Pharm Sci. 2013;48(4):680-8.

7. Fonteyne M, Vercruysse J, Diaz DC, Gildemyn D, Vervaet C, Remon JP, et al. Real-time assessment of critical quality attributes of a continuous granulation process. Pharm Dev Technol. 2013;18(1):85-97.

8. Ward HW, Blackwood DO, Polizzi M, Clarke H. Monitoring blend potency in a tablet press feed frame using near infrared spectroscopy. J Pharm Biomed Anal. 2013;80(Supplement C):18-23.

9. Alam MA, Shi Z, Drennen JK 3rd, Anderson CA. In-line monitoring and optimization of powder flow in a simulated continuous process using transmission near infrared spectroscopy. Int $\mathrm{J}$ Pharm. 2017;526(1-2):199-208.

10. Fonteyne M, Soares S, Vercruysse J, Peeters E, Burggraeve A, Vervaet $\mathrm{C}$, et al. Prediction of quality attributes of continuously produced granules using complementary pat tools. Eur J Pharm Biopharm. 2012;82(2):429-36.

11. Pawar P, Wang Y, Keyvan G, Callegari G, Cuitino A, Muzzio F. Enabling real time release testing by NIR prediction of dissolution of tablets made by continuous direct compression (CDC). Int $\mathrm{J}$ Pharm. 2016;512:96-107.

12. Orr J, Reid G. An introduction to process analytical technology. Pharmaceutical Online. June 17. Accessed May 2020. https:// www.pharmaceuticalonline.com/doc/an-introduction-to-processanalytical-technology-0001

13. Alam M, Drennan J, Anderson C. Designing a calibration set in spectral space for efficient development of an NIR method for tablet analysis. J Pharm Biomed Anal. 2017;145:230-9.

14. Shi Z, Hermiller J, García Muñoz S. Estimation of mass-based composition in powder mixtures using extended iterative optimization technology (EIOT). AICHE J. 2018;65(1):87-98.

15. ICH Q12 - Technical and regulatory considerations. 19-Nov-2019. Accessed 14-Sep-2020. https://database.ich.org/sites/default/files/ Q12_Guideline_Step4_2019_1119.pdf.

16. Mandenius C, Gustavsson R. Mini-review: soft sensors as means for PAT in the manufacture of bio-therapeutics. J Chem Technol Biotechnol. 2014;90(3):215-27.

17. Engisch WE, Muzzio FJ. Loss-in-weight feeding trials case study: pharmaceutical formulation. J Pharm Innov. 2014;10(1):56-75.

Publisher's Note Springer Nature remains neutral with regard to jurisdictional claims in published maps and institutional affiliations. 